\title{
Editorials
}

\section{Realising the potential of Improving Access to Psychological Therapies for older adults}

Depression and anxiety are significant problems for older adults and for those with chronic health problems." Social isolation and loneliness are significant risk factors for depression, ${ }^{2}$ and are particularly relevant to the older adult population in the UK, ${ }^{3,4}$ The COVID-19 pandemic and public health measures have restricted physical access of many to their social networks, ${ }^{5}$ and to faceto-face consultations. This is likely to have disproportionately affected the mental health of older adults and those with chronic health problems.

For depression and anxiety with mild-moderate functional impairment, psychoeducation and psychological therapies are recommended as first-line therapeutic options by the National Institute for Health and Care Excellence (NICE) evidence-based guidelines under distinct stepped-care approaches.7.8 The Improving Access to Psychological Therapies (IAPT) service was launched across England in 2008 in response to demand for more access to evidencebased psychoeducation and psychological therapies in the NHS.? There have been different initiatives to increase access to psychological therapies in Scotland, Wales, and Northern Ireland. ${ }^{10}$ Recent national datasets for England indicate that $>87 \%$ of patients who begin treatment with IAPT wait no longer than 6 weeks from their referral. ${ }^{11}$ However, there are regional variations, and waiting times for certain treatments are likely to be longer. Older adults have equal success rates with therapy compared with the rest of the population and are more likely to continue to engage with IAPT during treatment. ${ }^{12-14}$ However, under-represented groups among those accessing IAPT services or entering treatment include older adults (patients aged $\geq 65$ years), as well as those from lower incomes and ethnic minority groups, ${ }^{13-16}$ all of whom have been disproportionately impacted by COVID-19.

\section{ACCESSING PSYCHOLOGICAL THERAPIES}

Engagement is important for psychological therapies to be effective; ${ }^{17}$ therefore, IAPT typically encourage primary care to signpost patients to their services. This allows patients to self-refer at a time when they are prepared to engage with the therapeutic process. A minority of patients, for whom signposting and encouragement to self-refer would not be appropriate, are directly referred by GPs. Though there are benefits to this model,

"Recent national datasets for England indicate that $>87 \%$ of patients who begin treatment with IAPT wait no longer than 6 weeks from their referral.

there are additional barriers to self-referral in lower-income groups and older adults, who may need more support in order to selfrefer. ${ }^{18,19}$ Of those diagnosed with depression in primary care, there do not appear to be differences in direct referrals according to deprivation; however, fewer direct referrals to IAPT are made for older adults when compared with other age groups. ${ }^{20}$ Psychological therapies are discussed less with older adults as a management option for depression compared with younger adults, ${ }^{2}$ which is likely to reflect to both clinician and patient perspectives. ${ }^{19,22}$ Patient perspectives may include prioritising physical health problems, mental-health stigma, previous negative experiences with psychological therapists, the perception that depression is a normal part of ageing, and that services will not appreciate cultural experiences. ${ }^{19}$ Clinician perspectives may include the idea that they are primarily pharmacological prescribers, that depression is a normal response to age-related comorbidities, and that older adults are less likely to want to articulate depression, or to engage with or benefit from psychological therapies.22

Barriers to help-seeking and accessing psychological therapies can be categorised into system, patient, and clinician factors. Location offered for therapy sessions should not be a system barrier as IAPT typically see patients one-to-one at a general practice local to the patient or via telephone (especially during COVID-19). Although IAPT do not offer home visits, they do adapt sessions where appropriate to make therapy accessible to patients (for example, by offering telephone sessions). Most services also offer therapy in another language through interpreters. ${ }^{16}$ One system factor that does appear to be a barrier to help-seeking in some groups is the selfreferral model of access. ${ }^{18}$ This suggests that more equitable access can be achieved if older adults and those from lower socioeconomic groups are given extra support to access services either through direct referrals (when it is in a patient's best interest to do sol or greater GP endorsement of self-referral. ${ }^{19}$ Time constraints on consultations with GPs and advanced nurse practitioners may also be a greater barrier to help-seeking for older adults, who may put their physical health before their mental health, are more likely to have multiple physical health problems, and may require more information about what therapy may involve. ${ }^{19}$ Though the sustained pressures on primary care are a significant limiting factor, primary care clinicians can overcome this barrier by providing older adults with multiple conditions more time to consult and by using this time to initiate conversations with older adults about their mental health. This is arguably even more important given the increased proportion of telephone consultations recently, which reduce the opportunity to identify visual cues suggestive of mental health problems. ${ }^{23}$

\section{\#TALKINGHELPS}

Other barriers to help-seeking are the perspectives of patients and clinicians, and overcoming these requires both challenging the assumptions of clinicians and patients, and encouraging older adults to talk about their mental health. In 2020, NHS England and Age UK launched a joint campaign, \#TalkingHelps, to promote IAPT to older adult populations. ${ }^{24,25}$ The campaign has three key messages:

\section{Start the conversation: talking helps.}

2. Depression/anxiety are not just natural parts of ageing. It's not just your age'

3. Better mental health can mean better physical health. Don't put off your mental health.

The campaign realises the potential of GPs in overcoming barriers to help-seeking and the inequitable access to psychological therapies, and aims to support them in tackling this issue. It encourages doctors and other healthcare professionals to begin conversations with older adults about the 


\section{“In 2020, NHS England and Age UK launched a joint campaign, \#TalkingHelps, to promote IAPT to older adult populations.}

importance of their mental health and to consider signposting or referring older adults to IAPT. There is a range of resources for practices including posters, videos, and a guide that can be given to patients.

\section{THE ROLE OF GENERAL PRACTICE}

Though a complex combination of system, clinician, and patient factors have been implicated in the inequality of uptake of psychological therapy (such as the NHS England IAPT service), evidence suggests that there is potential for primary care clinicians to improve equity of access to psychological therapies through increased discussions and endorsement of selfreferral to services, ${ }^{21}$ and direct referrals. ${ }^{20}$
Primary care is well placed to initiate conversations with older adults about their mental health and signpost to psychological therapies. Given the effects of COVID-19 on social contact and the increase in telephone consultations, as well as the inherent risks associated with the prescribing of psychotropic medications in those with comorbidities and polypharmacy, it is particularly important that primary care continues to be proactive in promoting mental wellbeing for older adults.

\section{Jean-Pierre Laake,}

Postdoctoral Researcher and Medical Student,

Warwick Medical School, University of Warwick. Coventry.

\section{ADDRESS FOR CORRESPONDENCE}

\section{Jean-Pierre Laake}

Warwick Medical School, University of Warwick, Coventry CV4 7AL, UK

\section{Email: J.Laakeawarwick.ac.uk} aJPSLaake

\section{Nadia Majeed,}

Senior Psychological Wellbeing Practitioner, Coventry \& Warwickshire Partnership NHS Trust, Coventry.

\section{Kate Walters}

Clinical Professor of Primary Care and Epidemiology, Department of Primary Care and Population Health, University College London, London.

\section{Provenance}

Commissioned; externally peer reviewed.

\section{Competing interests}

The authors have declared no competing interests.

DOI: https://doi.org/10.3399/bjgp21X714365

\section{REFERENCES}

1. Rodda J, Walker Z, Carter J. Depression in older adults. BMJ 2011; 343: d5219.

2. Santini ZI, Jose PE, Cornwell EY, et al. Social disconnectedness, perceived isolation, and symptoms of depression and anxiety among older Americans (NSHAP): a longitudinal mediation analysis. Lancet Public Health 2020; 5(1): e62-e70.

3. Victor CR, Bowling A. A longitudinal analysis of loneliness among older people in Great Britain J Psychol 2012; 146(3): 313-331.

4. Office for National Statistics. General Lifestyle Survey 2011. London: ONS, 2013

5. Department of Health \& Social Care, Public Health England. Guidance on shielding and protecting people who are clinically extremely vulnerable from COVID-19. London: DHSC \& PHE, 2020.

6. Razai MS, Oakeshott $\mathrm{P}$, Kankam H, et al. Mitigating the psychological effects of social isolation during the covid-19 pandemic. BMJ 2020; 369: m1904.

7. National Institute for Health and Care Excellence. Depression in adults: recognition and management. CG90. London: NICE, 2009.

8. National Institute for Health and Care Excellence. Generalised anxiety disorder and panic disorder in adults: management. CG113. London: NICE, 2019.

9. NHS England. Adult Improving Access to Psychological Therapies programme. London: NHS England, 2019.
10. Blane DN, Williams C, Morrison J, et al. Psychological therapies in primary care: a progress report. Br J Gen Pract 2014; DOI: https://doi.org/10.3399/bjgp14X682117.

11. NHS Digital. Psychological therapies: reports on the use of IAPT services, England - February 2020. Final including reports on the IAPT pilots. NHS Digital, 2020.

12. Gould RL, Coulson MC, Howard RJ. Cognitive behavioral therapy for depression in older people: a meta-analysis and meta-regression of randomized controlled trials. J Am Geriatr Soc 2012; 60(10): 1817-1830.

13. NHS Digital. Psychological therapies: annual report on the use of IAPT services 2016-17. London: NHS Digital, 2017.

14. NHS Digital. Psychological therapies: annual report on the use of IAPT services 2017-18. London: NHS Digital, 2018.

15. Baker C. Mental health statistics for England: prevalence, services and funding. London: House of Commons Library, 2018.

16. Royal College of Psychiatrists. Report of the Second Round of the National Audit of Psychological Therapies (NAPT). London: Healthcare Quality Improvement Partnership, 2013.

17. Papworth M, Marrinan T, Martin B, et al Low intensity cognitive behaviour therapy: a practitioner's guide. Los Angeles, CA: SAGE Publications, 2013.

18. Thomas F, Hansford L, Ford J, et al. How accessible and acceptable are current GP referral mechanisms for IAPT for low-income patients? Lay and primary care perspectives. J Ment Health 2019; 4: 1-6.

19. Nair P. Bhanu C. Frost R, et al. A systematic review of older adults' attitudes towards depression and its treatment. Gerontologist 2020; 60(1): e93-e104.

20. Walters K, Falcaro M, Freemantle N, et al. Sociodemographic inequalities in the management of depression in adults aged 55 and over: an analysis of English primary care data. Psychol Med 2018; 48(9): 1504-1513.

21. Laake JP, Parratt J, Majeed N. Improving Access to Psychological Therapies for older adults: auditing management of newly diagnosed mild and moderate depression in six general practices within the Birmingham and Solihull CCG. Postgrad Med J 2020; DOI: 10.1136/postgradmedj-2019-136996.

22. Frost R, Beattie A, Bhanu C, et al. Management of depression and referral of older people to psychological therapies: a systematic review of qualitative studies. Br J Gen Pract 2019; DOI: https://doi.org/10.3399/bjgp19X701297.

23. Mulvihill C, Cooper J, Pavey J, Laake JP. Remote consultations in primary care during the COVID-19 pandemic: student perspectives. Postgrad Med J 2020; DOI: 10.1136/ postgradmedj-2020-139149.

24. Age UK. Your mind matters. Age UK, 2020.

25. NHS England. Older people encouraged to ditch 'stiff upper lip' approach to mental ill health. London: NHS England, 2020. 